\title{
Le Congrès général de Strasbourg 2015
}

Le XXIII ${ }^{\mathrm{e}}$ congrès de la Société Française de Physique, organisé par la section Alsace, s'est déroulé à Strasbourg du 24 au 28 août 2015. Les participants ont été accueillis dans les locaux rénovés du campus de l'université qui se trouve être, hors Paris, le dernier encore situé en centre-ville.

Ce congrès a tout particulièrement bénéficié du soutien de l'université de Strasbourg, qui, outre une aide financière de son programme IdEx (Initiative d'excellence), a gracieusement prêté ses locaux. Nous remercions également, pour leurs soutiens financiers et matériels, la ville et l'Eurométropole de Strasbourg, le conseil régional d'Alsace, les instituts et la délégation régionale Alsace du CNRS, le Labex NIE (Nanostructures en Interaction avec leur Environnement) et la Fondation pour la recherche en chimie.
Ce texte maintient la tradition de la rédaction du compte-rendu du congrès général fait par les organisateurs, bien qu'un regard extérieur serait probablement plus objectif. Indiquons pour commencer que le millésime 2015 du congrès a parfaitement rempli sa fonction première, qui était de braquer le projecteur sur l'activité en physique d'un territoire particulier. Toutes les composantes alsaciennes ont contribué à son succès : les universités de Strasbourg et de Mulhouse, ainsi, bien entendu, que l'ensemble des laboratoires de physique du site. Rien ne se serait fait bien sûr si les enseignants-chercheurs et les chercheurs ne s'étaient pas si fortement mobilisés pour présenter leur activité sous son meilleur jour. Le congrès a suscité un intérêt marqué auprès des enseignants du secondaire qui avaient déjà participé à la «Journée de la
Lumière " organisée par la "Maison de la science en Alsace " en mars 2015, dans le cadre de l'" Année de la Lumière ".

Il convient de remercier ici vivement les collègues et les étudiants qui ont bénévolement consacré une partie de leur temps précieux à assurer le bon fonctionnement de l'événement : accueil, fléchage, affichage, support technique des sessions plénières et des colloques. Nous avons la chance à Strasbourg de bénéficier des compétences et de l'expérience de la " cellule congrès » de l'université qui a, une fois encore, soulagé les organisateurs en assurant l'inscription et l'accueil des participants et des conférenciers et en préparant en amont toute l'intendance qui, quand elle est réussie, garantit la qualité de la conférence.

Les échanges avec Daniel Estève, président du comité scientifique du congrès, et

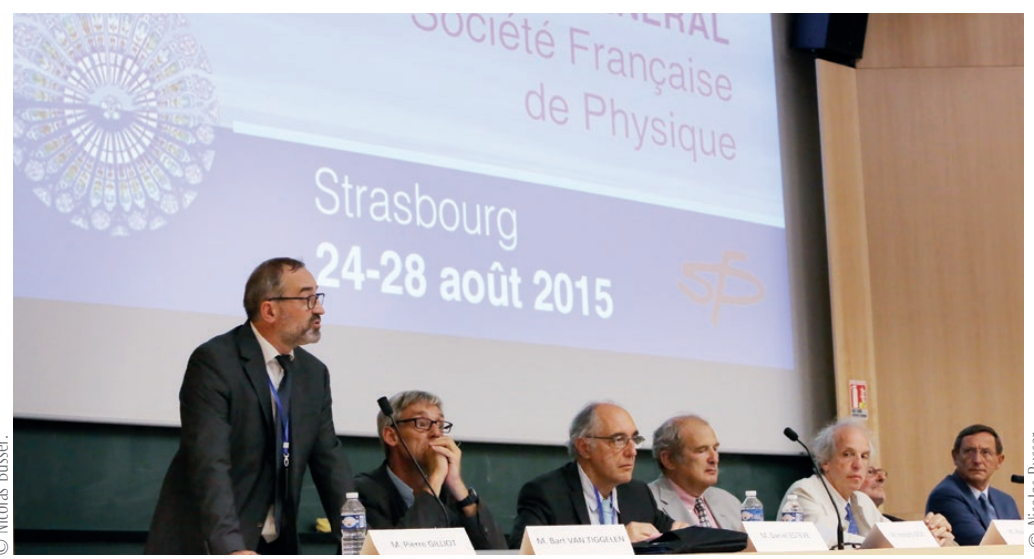

1. L'ouverture du congrès. De gauche à droite : Pierre Gilliot (président du comité local), Bart van Tiggelen (CNRS-INP), Daniel Estève (président du comité scientifique), François Loss (conseiller municipal de Strasbourg), Alain Beretz (président de l'Université de Strasbourg), Alain Fontaine (président de la SFP) et Robert Herrmann (président de l'Eurométropole de Strasbourg).

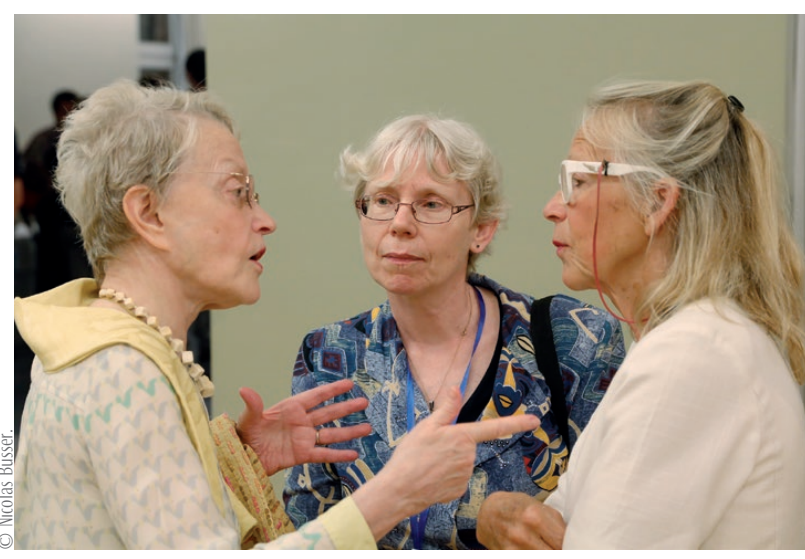

2. Discussion animée pendant le colloque « Femmes et physique ». De gauche à droite : Jacqueline Chénieux-Gendron (EHESS), Véronique Pierron-Bohnes (IPCMS) et Françoise Brochard (Institut Curie). 


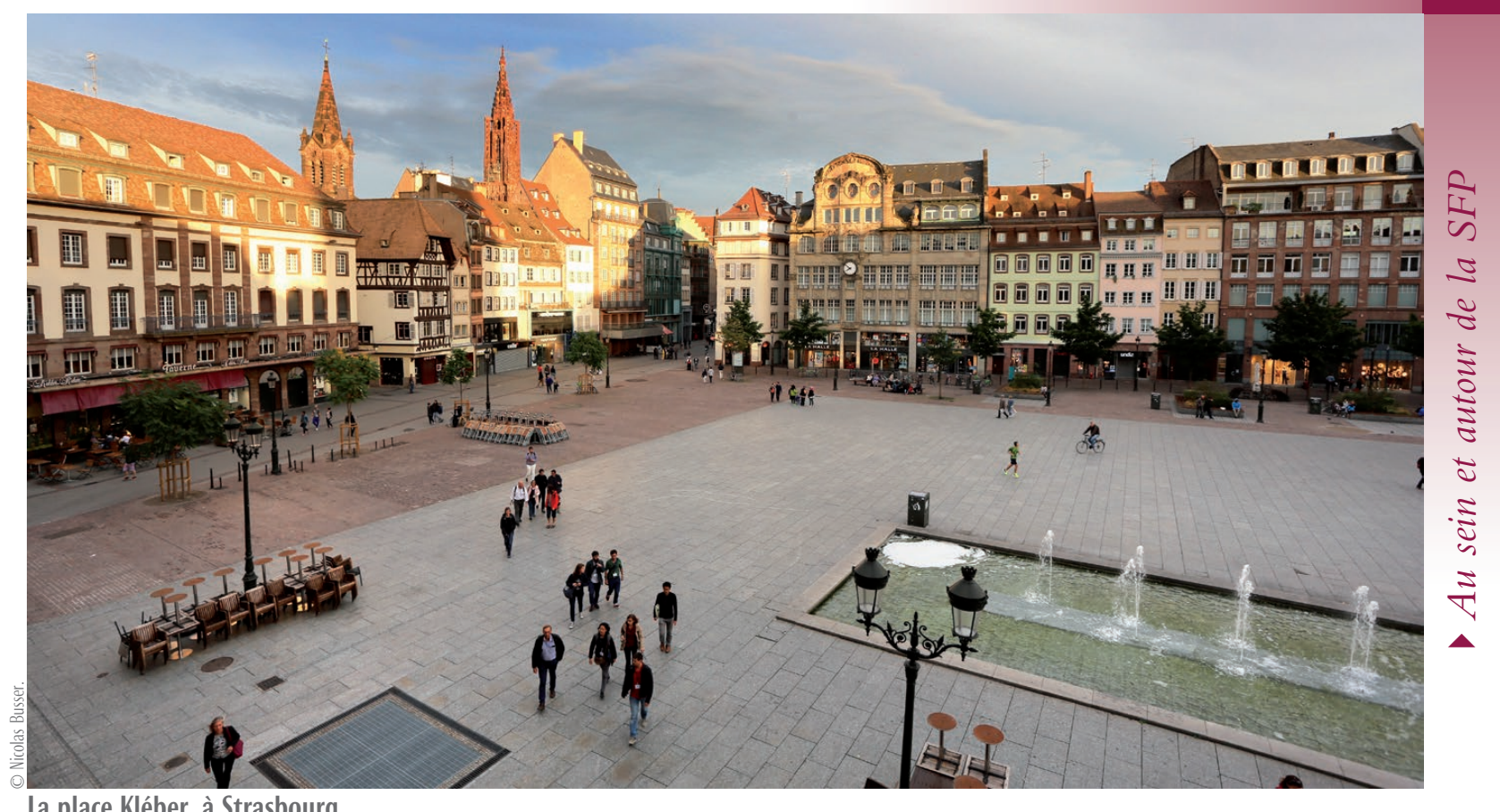

Alain Fontaine, président de la SFP, ont été marqués par le pragmatisme et l'efficacité des actions de chacun. Mayline Gautié a suivi depuis le siège à Paris le déroulement de l'organisation pour coordonner les actions, et elle a œuvré sur nombre de points pratiques.

Le XXIII ${ }^{\mathrm{e}}$ congrès général de la SFP a accueilli 515 participants qui ont délivré 298 conférences et 128 affichages; il se situe favorablement dans la lignée des congrès qui ont précédé, malgré la crainte des organisateurs de voir une certaine désaffection causée par la date choisie. Ce choix de date a en fait été plutôt favorable à une bonne participation des étudiants qui n'avaient plus à se préoccuper de préparer les conférences internationales de l'été. Cette date a aussi été perçue positivement par les enseignants de physique du secondaire, pour qui le congrès a servi de pré-rentrée récréative.

$12 \%$ des inscrits venant de treize autres pays ont souligné l'attraction exercée par le congrès sur les physiciens francophones à l'étranger. Il convient de noter une importante délégation d'étudiants de l'université d'Annaba en Algérie. Au-delà de la forte participation attendue des Alsaciens au congrès, la géographie de la répartition des participants sur le territoire national est le pur reflet de la distribution des grandes institutions de recherche en physique. Par contraste, on note l'absence totale de participation des départements et territoires d'outre-mer.

Pour ce qui concerne la répartition par genres, la proportion des physiciennes parmi les inscrits est faible (12\%), inférieure à celles des femmes à la SFP (18\%), dans les principaux organismes de recherche et l'université ( $22 \%)$. Les femmes ont cependant été très présentes à tous les niveaux du congrès comme le montrent, par exemple, la proportion de femmes invitées en conférences plénières ou semiplénières, ou ayant contribué par une présentation par affiche (28\%).

Et pour terminer, il faut aussi noter que les industriels ne se sont pas battus pour obtenir un stand. Seuls JEOL (microscopes électroniques...), Hamamatsu (optronique) et l'éditeur Springer ont fait le déplacement à Strasbourg. À une époque où il est de bon ton d'encourager le transfert technologique ("from ideas to innovation, comblons la vallée de la mort ") par la création de start-up, une seule est venue : RBnano (revêtements et traitements de surface).

\〉

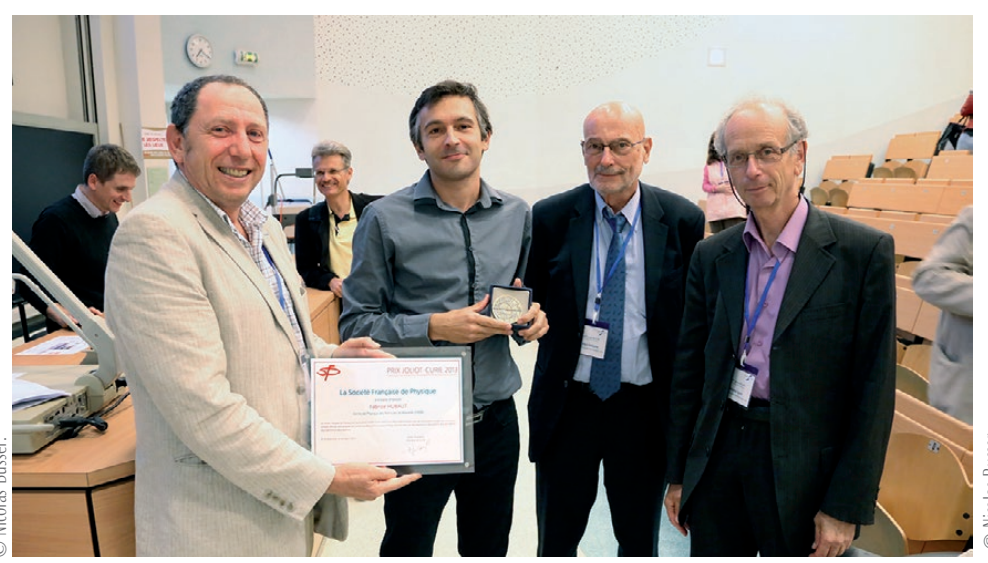

3. Remise du prix Joliot-Curie 2013. De gauche à droite : Guy Wormser (président de la division champs et particules de la SFP), Fabrice Hubaut, Alain Fontaine et Michel Spiro (vice-président de la SFP).

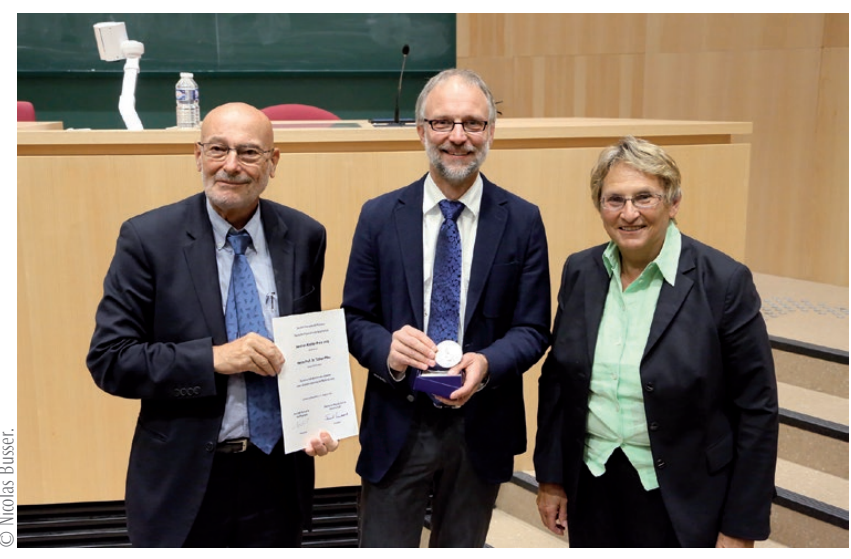

4. Remise du prix Gentner-Kastler 2015. De gauche à droite : Alain Fontaine, Tilman Pfau et S.G. Zicknagl (DPG). 


\section{\〉 \\ Les conférences plénières et semi-plénières}

Ces conférences, données par les meilleurs spécialistes dans leur domaine, ont permis de dresser un tableau clair et concis des champs disciplinaires de la physique les plus en pointe aujourd'hui. Et l'on vit même quelques authentiques représentants du grand public s'installer dans l'amphi à l'heure de la conférence sur la théorie des cordes qui leur était destinée !

La conférence de Michèle Leduc sur l'éthique en science et les bonnes pratiques en recherche, a reçu un accueil attentif par sa résonance avec quelques récentes affaires de mauvaise conduite ayant émaillé et terni des disciplines proches de la nôtre.

\section{Les colloques de spécialité}

Les colloques de spécialité, au nombre de 29, ont permis d'approfondir les grands sujets effleurés en séance plénière, mettant un accent particulier sur l'optique, "Année de la Lumière " oblige, et les domaines interdisciplinaires.

Enfin, les colloques «Physique \& Société » ont été l'occasion d'échanger sur divers aspects de la vie de la physique en France : - la situation des femmes en physique : les dernières statistiques, l'amplification des stéréotypes filles-garçons à l'école, les moyens d'action possibles ;

- l'emploi des jeunes chercheurs-euses : quelle stratégie adopter pendant et après la thèse ? quels sont les parcours possibles?

- l'impact du changement des programmes de physique au lycée dans l'enseignement supérieur ;

\section{Pierre Gilliot (pierre.gilliot@ipcms.unistra.fr) et Charles Hirlimann Institut de physique et de chimie des matériaux de Strasbourg (IPCMS) Comité d'organisation local du $23^{\mathrm{e}}$ congrès général de la SFP.}

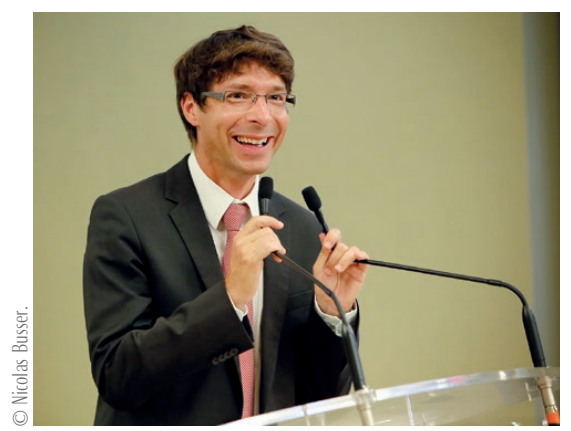

5. Nicolas Matt, adjoint au maire de Strasbourg, reçoit le congrès dans les salons de l'Aubette, place Kléber.

- la dissémination de la culture scientifique : actions régionales, exemples de dispositifs de culture scientifique à destination des collèges et lycées..

- les bonnes pratiques de la recherche.

\section{Remises de prix}

- Lundi 24 août à 18 h30 : en clôture du colloque LHC, remise du prix JoliotCurie 2013 à Fabrice Hubaut (Centre de physique des particules de Marseille) pour sa contribution exceptionnelle à l'expérience ATLAS au CERN (fig. 3).

- Mardi 25 août à 15h30 : dans le cadre du colloque Plasmas, remise du prix René Pellat 2015 à Stéphane Cuynet (Groupe de recherche sur l'énergétique des milieux ionisés, Orléans), pour ses travaux sur l'élaboration de piles à combustible par procédé plasma.

- Mercredi 26 août à 9 h30 : remise du prix Gentner-Kastler 2015 (prix décerné par la SFP et la DPG, Société allemande de physique) à Tilman Pfau (Université de Stuttgart) pour ses travaux sur les gaz ultra-froids (fig. 4).

\section{Les activités sociales}

Les participants ne pouvaient pas venir à Strasbourg sans goûter quelques-unes des spécialités qui font la renommée de la gastronomie de la région. Ils en ont eu l'occasion lors du buffet dinatoire offert par la ville de Strasbourg (fig. 5), et aussi à l'occasion du banquet (fig. 6) qui s'est tenu dans la célèbre maison Kammerzell, place de la cathédrale Notre-Dame.

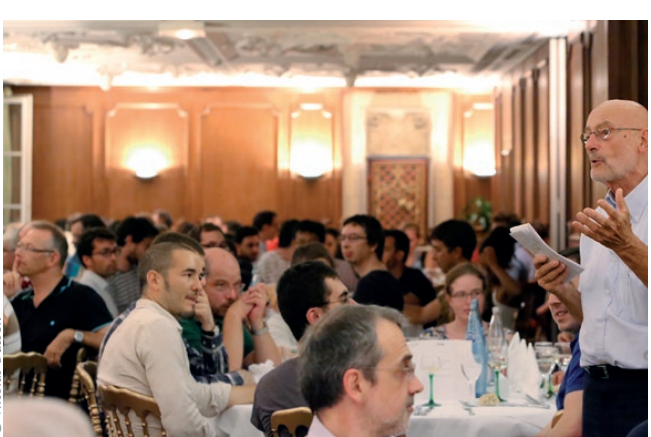
à l'ouverture du banquet.
6. Discours du président de la SFP, Alain Fontaine,

\section{Liste des conférences}

\section{Conférences plénières}

- Far-field optical nanoscopy: principles and recent advancements, Stefan Hell (prix Nobel de chimie 2014).

- At the frontier between ultrafast optics and magnetism, Jean-Yves Bigot.

- Des noyaux " exotiques "à la lumière extrême : les nouvelles frontières de la physique nucléaire, Sydney Gales.

- Les gaz ultra-froids : un monde quantique entre physique atomique et matière condensée, Jean Dalibard.

- Les bonnes pratiques de la recherche, Michèle Leduc.

- Physique au LHC : passé, présent, futur, Isabelle Wingerter.

- Strongly interacting Rydberg gases in thermal vapor cells, Tilman Pfau.

- Mission cométaire Rosetta, Wlodek Kofman.

- Hybrid light-matter states, Thomas Ebbesen.

- Entangled active matter: from ants to living cells, Françoise Brochard-Wyart.

- Mille ans d'optique, 50 ans de solitons, John Dudley.

- Les résultats du LHC sur le boson de Higgs, Guillaume Unal.

- Vitrimères, une nouvelle classe de matériaux organiques, Ludwik Leibler.

- Spin-orbitronics, a new direction for spintronics,

Albert Fert (Prix Nobel de physique 2007).

\section{Conférences semi-plénières}

- Interstellar PAHs: a challenge for molecular physicists, Christine Joblin.

- Électronique de spin à l'échelle de l'électron unique, Tristan Meunier.

- Dynamique en temps réel d'un matériau sous l'impact d'un pulse laser : disséquer, sélectionner, agir, modifier, Hervé Cailleau.

- Optique quantique avec des émetteurs uniques à l'état solide, Pascale Senellart.

- Physique hors d'équilibre : quelques progrès récents, Kirone Mallick.

- L'accélération laser-plasma, une piste pour les futurs accélérateurs, Brigitte Cros.

Une conférence à destination du grand public, sur un sujet difficile

- Principe d'équivalence et gravité quantique, Pierre Vanhove. 